\title{
Evolution and ecology, after the malaria genomes
}

\author{
Jacobus C. de Roode and Andrew F. Read \\ Institute of Cell, Animal and Population Biology, University of Edinburgh, King's Buildings, West Mains Road, Edinburgh EH9 3JT, UK
}

\begin{abstract}
The first of the three genomes responsible for malaria was published last year; with the recent and much hyped publication of parasite and vector genomes in Nature and Science respectively, the life-cycle triad is now complete. The genomes and associated papers demonstrate that evolutionary biologists and ecologists have much to contribute to malaria control - and to the unravelling of some very interesting biology.
\end{abstract}

The recently announced sequencing of the genomes of the most virulent human malaria parasite Plasmodium falciparum [1], its most important vector Anopheles gambiae [2], and that of the rodent malaria Plasmodium yoelii yoelii [3], has rightly engendered much enthusiasm among malaria researchers. It is now possible, for instance, to search the full genomes for new drug and vaccine targets, and hundreds of candidates are likely to be found. Quite when this will impact on malaria control is, however, an open question. Novel drugs are certainly required. But testing the candidate vaccines that we already have is currently rate-limiting and, even if the candidates now under trial were actually to work, regulatory bureaucracy means it would be $10-15$ years before they could be deployed (by which time another ten million people will have died). The substantial human [4] and economic [5] costs of malaria will be with us for some time.

Malaria research has been dominated by biomedical science, but the failure of that science to control malaria is largely because of evolution and ecology. Both parasite and vector have evolved around drugs; vaccines are not in use because the parasite possesses adaptations to promote somatic evolution; and the robust ecology of the vector and parasite challenge standard public health approaches. The time is ripe for ecologists and evolutionists to join biomedical researchers in the post-genomic malaria world. Not only will this solve many emerging evolutionary puzzles, but it will also be an absolute necessity to solve the ecological questions that, for example, genetically modified (GM) mosquitoes will pose. Here, we summarize some of the opportunities that emerge from the Nature and Science issues that contained the genomes.

\section{Evolution of antigenic variation}

One puzzle is species divergence in antigenic variation genes. Comparison of the $P$. falciparum and $P$. y. yoelii genomes reveals that the two species use nonhomologous gene families to change their antigenic properties and thus escape immune recognition [6]. The $P$. falciparum genome

Corresponding author: Jacobus C. de Roode (j.de.roode@ed.ac.uk). contains 59 var, 149 rif and 28 stevor genes [1]. Rif and stevor genes are probably involved in antigenic variation, but var genes definitely are. They encode proteins on the red blood cell membrane, where they are involved in adhesion to host endothelial receptors [1], thus making them a direct target for the immune system. An individual parasite only transports one var protein to its cell surface, but individuals in a clonal lineage can express different proteins and hence evade the immune system.

The rodent malaria P. y. yoelii does not possess homologues of var, rif or stevor genes [3], in spite of great overall gene synteny between the two species: of $\sim 5300 P$. falciparum genes, $>3300$ P. y. yoelii orthologues were found. A probable candidate for $P$. $y$. yoelii antigenic variation genes is the yir gene family, containing no less than 838 genes. They are homologous with the vir gene family in the human malaria parasite $P$. vivax, encoding proteins that are immunovariant in natural infections and that are likely to be involved in antigenic variation and immune evasion [3].

Differences in the gene families involved could mirror differences in the strategies adopted. Even so, why would these species differ in the genetic basis of antigenic variation? Presumably they share a common ancestor that also had to deal with acquired immunity. The differences cannot be caused simply by adaptations to different hosts, because $P$. $y$. yoelii and $P$. vivax have homologous immune evasion genes. Searches for homologous gene families in other rodent and primate malarias, as well as those in birds and lizards, will be very interesting, especially given that more rigorous Plasmodium phylogenies are beginning to emerge [7]. The nature of selection on diversity-generating mechanisms has long been the subject of conventional evolutionary biology. With the genomes in our hands, it is now time to apply this expertise to infectious disease [6].

\section{Evolution of multigene families}

Antigenic variation genes are not the only genes to occur in gene families. In fact, both the Plasmodium and Anopheles genome show many expansions of genes and gene families, possibly providing parasite and vector with a great adaptive ability.

The Anopheles genome contains many gene families, and these are often highly divergent from those in Drosophila melanogaster. For instance, the 242 Anopheles genes from 18 families implicated in innate immunity show marked diversification from Drosophila and a deficit of orthologues [8]. Of the 79 Anopheles G protein-coupled receptors identified as candidate odorant receptors, 27 do not have close Drosophila relatives [9]. It is tempting to attribute these genome-wide differences to selection 
pressures from different food sources. But why should detecting rotting fruit or humans require radically different genetic control? And why should the fruit- and blood-borne pathogens require such different immune control? Has gene proliferation been a cause or a consequence of the adaptive plasticity that has the malaria parasite and its vector resilient in the face of 100 years of medical onslaught?

\section{Vector behaviour and physiology}

Malaria parasites decrease vector fecundity [10] and increase biting rate and blood-meal size [11]. These alterations could be adaptive for the parasite (e.g. reallocation from reproduction to parasite nutrition) or host (reallocation from reproduction to immune function), or adaptive for neither. Differentiating between these alternatives can be done by measuring the fitness consequences of the altered phenotypes for the players involved, but it is not always easy to manipulate these experimentally. A different approach is to determine which genes are running the show.

Holt et al. [2] present a study of blood-meal-fed and nonblood-meal-fed mosquitoes, and show that genes involved in egg melanisation and yolk and oocyte production were upregulated in blood-fed mosquitoes. A next step is to compare gene expression patterns of mosquitoes fed infected or uninfected blood. Alternatively, are there Anopheles-like genes in the malaria genome? These might be good candidates as host-manipulation genes.

One candidate gene involved in the decreased fecundity is one of 35 putative regulatory genes [12]. This gene is closely related to an Aedes aegypti homologue that is expressed in ovaries only during reproductive arrest and the first $24 \mathrm{hr}$ of a reproductive cycle when ecdysteroids are being synthesized. This receptor is possibly involved in hormonal regulation and physiological pathways that, in D. melanogaster and Caenorhabditis elegans, are involved in the link between longevity and fecundity. If this is the key regulatory switch between arrest/longevity and reproduction in An. gambiae, it could be the target mechanism of any adaptive manipulation of the reproductive physiology of the mosquito by the parasite.

\section{Ecological challenges of GM mosquitoes}

In the past, malaria control has been achieved mainly by mosquito control [13]. With the increasing problems of insecticide resistance, many malaria researchers now focus their hopes on changing the genetic makeup of the mosquito to block transmission or to decrease mosquito fitness. The genomes will provide many candidate genes to accomplish this. Rapidly advancing technology has already made it possible to create transgenic mosquitoes that do not transmit malaria [14]. Indeed, we are now at a stage at which the technological challenges of manipulating genes in vivo are trivial compared to the ecological challenges of using this technology to control malaria [15].

There is room for a lot more thinking about which genes should be used and then how they should be driven into wild populations [15]. This is an area that has already seen significant contributions from evolutionary biologists [16]. But much more is needed, particularly to fill out our incomplete understanding of parasite transmission ecology [17]. For example, we need to know more about gene flow within and between mosquito populations. What are the fitness consequences of genetic modification of mosquitoes? And what counter evolution by the parasite will GM mosquitoes prompt? Scott et al. [17] argue that continued evaluation of GM technology will require release of GM mosquitoes on isolated oceanic or ecological islands that have been thoroughly characterized with respect to the genetic and ecological makeup of local mosquito vector populations and site-specific patterns of pathogen transmission and disease. That is a lot of untrendy 'muddy boots' ecology.

\section{Outlook}

Clearly, the molecular biologists who make GM mosquitoes will need ecologists to tackle these complicated ecological problems. In a time of increasingly big science, even the early findings from the post-genomic era show that readers of TREE will be as needed in malaria research as are readers of Trends in Genetics, Parasitology or Microbiology. Indeed, in an academic world where we know an enormous amount about banana-dwelling flies, one could argue that a focus by evolutionary biologists and ecologists on an organism that is at least as interesting as a fruit fly is long overdue. Research funding opportunities are arguably the only benefit of the catastrophic death toll from malaria.

\section{References}

1 Gardner, M.J. et al. (2002) Genome sequence of the human malaria parasite Plasmodium falciparum. Nature 419, 498-511

2 Holt, R.A. et al. (2002) The genome sequence of the malaria mosquito Anopheles gambiae. Science 298, 129-149

3 Carlton, J.M. et al. (2002) Genome sequence and comparative analysis of the model rodent malaria parasite Plasmodium yoelii yoelii. Nature $419,512-519$

4 Breman, J.G. (2001) The ears of the hippopotamus: manifestations, determinants, and estimates of the malaria burden. Am. J. Trop. Med. Hyg. 64, 1-11

5 Sachs, J. and Malaney, P. (2002) The economic and social burden of malaria. Nature $415,680-685$

6 Frank, S.A. (2002) Immunology and Evolution of Infectious Disease, Princeton University Press

7 Perkins, S.L. and Schall, J.J. (2002) A molecular phylogeny of malarial parasites recovered from cytochrome $b$ gene sequences. J. Parasitol. $88,972-978$

8 Christophides, G.K. et al. (2002) Immunity-related genes and gene families in Anopheles gambiae. Science 298, 159-165

9 Hill, C.A. et al. (2002) G protein coupled receptors in Anopheles gambiae. Science $298,176-178$

10 Hurd, H. et al. (1995) Interactions between bloodfeeding, fecundity and infection in mosquitos. Parasitol. Today 11, 411-416

11 Koella, J.C. (1999) An evolutionary view of the interactions between anopheline mosquitoes and malaria parasites. Microbes Infect. 1, 303-308

12 Riehle, M.A. et al. (2002) Neuropeptides and peptide hormones in Anopheles gambiae. Science 298, 172-175

13 Spielman, A. and D'Antonio, M. (2001) Mosquito. A Natural History of our most Persistent and Deadly Foe, Faber and Faber

14 Ito, J. et al. (2002) Transgenic anopheline mosquitoes impaired in transmission of a malaria parasite. Nature $417,452-455$

15 Alphey, L. et al. (2002) Malaria control with genetically manipulated insect vectors. Science $298,119-121$

16 Turelli, M. and Hoffmann, A.A. (1999) Microbe-induced cytoplasmic incompatibility as a mechanism for introducing transgenes into arthropod populations. Insect Mol. Biol. 8, 243-255

17 Scott, T.W. et al. (2002) The ecology of genetically modified mosquitoes. Science 298, 117-119

0169-5347/02/\$ - see front matter $(\subset 2002$ Elsevier Science Ltd. All rights reserved. PII: S0169-5347(02)00058-7 